\title{
Demonstration of passive plasma lensing of a laser wakefield accelerated electron bunch
}

\author{
S. Kuschel, ${ }^{1,2}$ D. Hollatz, ${ }^{1,2}$ T. Heinemann, ${ }^{3}$ O. Karger, ${ }^{3}$ M. B. Schwab, ${ }^{1}$ D. Ullmann, ${ }^{1}$ \\ A. Knetsch, ${ }^{3}$ A. Seidel, ${ }^{1}$ C. Rödel,${ }^{1,4}$ M. Yeung, ${ }^{2}$ M. Leier, ${ }^{1}$ A. Blinne, ${ }^{2,5}$ H. Ding, ${ }^{6}$ T. Kurz, ${ }^{6}$ \\ D. J. Corvan, ${ }^{7}$ A. Sävert, ${ }^{1}$ S. Karsch, ${ }^{6}$ M. C. Kaluza, ${ }^{1,2}$ B. Hidding, ${ }^{8,3}$ and M. Zepf ${ }^{1,2,7}$ \\ ${ }^{1}$ Institute of Optics and Quantum Electronics, Friedrich-Schiller-University of Jena, \\ Max-Wien-Platz, 1, 07743 Jena, Germany \\ ${ }^{2}$ Helmholtz Institute Jena, Fröbelstieg 3, 07743 Jena, Germany \\ ${ }^{3}$ Institute for Experimental Physics, University of Hamburg, \\ Luruper Chaussee 149, 22761 Hamburg, Germany \\ ${ }^{4}$ SLAC National Accelerator Laboratory, 2575 Sand Hill Road, Menlo Park, California 94025, USA \\ ${ }^{5}$ Theoretisch-Physikalisches Institut, University of Jena, Max-Wien-Platz 1, 07743 Jena, Germany \\ ${ }^{6}$ Ludwig-Maximilians-Universität München, Am Coulombwall 1, D-85748 Garching, Germany \\ ${ }^{7}$ School of Mathematics \& Physics, Queens University, Belfast BT7 1NN, United Kingdom \\ ${ }^{8}$ SUPA, Department of Physics, University of Strathclyde, G4 ONG Glasgow, United Kingdom \\ (Received 15 January 2016; revised manuscript received 12 April 2016; published 20 July 2016)
}

\begin{abstract}
We report on the first demonstration of passive all-optical plasma lensing using a two-stage setup. An intense femtosecond laser accelerates electrons in a laser wakefield accelerator (LWFA) to $100 \mathrm{MeV}$ over millimeter length scales. By adding a second gas target behind the initial LWFA stage we introduce a robust and independently tunable plasma lens. We observe a density dependent reduction of the LWFA electron beam divergence from an initial value of $2.3 \mathrm{mrad}$, down to $1.4 \mathrm{mrad}(\mathrm{rms})$, when the plasma lens is in operation. Such a plasma lens provides a simple and compact approach for divergence reduction well matched to the mm-scale length of the LWFA accelerator. The focusing forces are provided solely by the plasma and driven by the bunch itself only, making this a highly useful and conceptually new approach to electron beam focusing. Possible applications of this lens are not limited to laser plasma accelerators. Since no active driver is needed the passive plasma lens is also suited for high repetition rate focusing of electron bunches. Its understanding is also required for modeling the evolution of the driving particle bunch in particle driven wake field acceleration.
\end{abstract}

DOI: 10.1103/PhysRevAccelBeams.19.071301

\section{INTRODUCTION}

Linear accelerators reaching energies in the range of hundreds of $\mathrm{MeV}$ to $\mathrm{GeV}$ are typically large devices, their size determined by accelerating fields in the range of tens of $\mathrm{MeV} / \mathrm{m}$. Focusing optics for these devices have been developed to high standards and operate on commensurate length scales. Alternatively, plasmas can support extremely large electric fields, which, for the common conditions of laser driven wakefield accelerators (LWFA), are typically in the range of hundreds of $\mathrm{GV} / \mathrm{m}$ [1-3]. Consequently extremely compact particle acceleration structures are possible leading to acceleration energies in excess of $\mathrm{GeV}$ taking place over $\mathrm{cm}$ length scales [4]. However, transportation, shaping and focusing of accelerated particle beams still require conventional structures such as quadrupole magnets and solenoids which are typically much larger

Published by the American Physical Society under the terms of the Creative Commons Attribution 3.0 License. Further distribution of this work must maintain attribution to the author $(s)$ and the published article's title, journal citation, and DOI. than the wakefield accelerator itself. The divergence of LWFA-generated electron beams is typically on the order of a few mrad $[5,6]$ and is thus relatively large compared to many radio frequency (rf) cavity based electron beams, although their emittances have comparable values. A similarly compact, sufficiently strong collimating lens would thus be a perfect complement to the laser-wakefield accelerator. A natural route toward such a focusing device would be to exploit the high field strengths in a plasma not only for accelerating but also for focusing the particle bunch. Therefore, plasma lenses have attracted notable interest in the literature because of their ability to provide compact beam collimation/focusing devices for energetic electron beams [7-9]. These are of particular interest for the rapidly developing field of laser plasma accelerators. However, LWFA beams depart significantly from the parameter range where these devices have previously been considered.

Possible applications of such a compact collimating device include beam shaping for transport or injecting an electron beam into a conventional undulator structure [10-12]. Others include the generation of dense, 
femtosecond positron beams [13] or bright, high-energy $\gamma$-ray beams [14,15] as well as enhancing the bunch density for Thomson backscattering sources [16-18]. Beyond these applications, understanding the plasma lens effect is in many ways important for beam shaping of LWFAgenerated electron bunches after the accelerator stage and also in the context of plasma wakefield accelerators (PWFA): For example, lensing during entry into a plasma can be used to match the transverse size of bunches inside the plasma wave to the focusing force to avoid beam oscillations [19]. The effect of plasma lensing is an integral part of proposed all-optical, hybrid plasma accelerators, in which electron bunches are generated in a LWFA stage and then subsequently used to drive a plasma wave via PWFA in a second stage. One configuration is a driverwitness scenario, which may lead to efficient energy transfer [20-22], whilst another especially attractive scenario is the "Trojan Horse" scheme, in which ultrahigh quality electron bunches may be generated via an underdense photocathode process [23]. Controlling the plasma lensing - an effect which is ubiquitous in these schemesis therefore of crucial importance.

The plasma lens effect is typically divided into the overdense $\left(n_{b} \ll n_{p}\right)$ and the underdense $\left(n_{b} \gg n_{p}\right)$ regime, where $n_{b}$ and $n_{p}$ denote the electron density in the bunch and the plasma respectively. In the underdense case, the bunch drives a strong plasma wave in the background plasma (even up to the blowout regime [24]) and the bunch electrons are focused due to the transverse electric fields of the plasma wave. In the overdense regime, the bunch's self-fields create only a small perturbation of the plasma density. In a freely propagating electron beam, the repulsive electric field is largely balanced by the focusing field of the self-generated magnetic field (the electric and magnetic forces of a bunch propagating through vacuum cancel out exactly in the limit of $v=c$ ). In the overdense regime the focusing force results from the plasma shielding the repulsive electric field of the bunch, leaving a net focusing force due to the magnetic field which pinches the bunch [25].

The plasma lens effect [26] has been experimentally demonstrated in the overdense regime [7,27-30] for low-energy $(<50 \mathrm{MeV})$, low-density bunches and plasma densities in the range of $10^{11}-10^{13} \mathrm{~cm}^{-3}$. In these experiments, the electron bunches were created by using $\mathrm{rf}$ accelerators and therefore the bunch duration was in the range of 10 picoseconds or longer. Here we present experimental data of passive lensing for electron bunches with a pulse duration which is around three orders of magnitude shorter than previously reported (6 fs of LWFA electron bunches reported by [31]).

We report the first demonstration of passive plasma focusing of a laser wakefield accelerated electron bunch resulting in an ultracompact, all-optical setup (in which the plasma lens is ionized using the LWFA driving laser).
"Passive" in this context means that the bunch self-focuses during its propagation through the plasma in contrast to an "active" plasma lens [32,33], where the fields of a pregenerated plasma structure-typically another wakefield, that is not driven by the bunch itself-focus the bunch, as recently demonstrated in [34]. With the passive plasma lens, described here, it is thus possible to focus an isolated electron bunch using a sufficiently dense plasma.

\section{EXPERIMENT}

The experiment presented here was conducted at the JETI 40 Ti:sapphire laser system at the University of Jena, delivering $\tau=28 \mathrm{fs}$ pulses at a central wavelength $\lambda_{0}=800 \mathrm{~nm}$ with $E=650 \mathrm{~mJ}$ on target. The laser pulses were focused by an $f / 12$ off-axis parabolic mirror to a spot size of $120 \mu \mathrm{m}^{2}$ (FWHM), resulting in a vacuum peak normalized amplitude of $a_{0}=\sqrt{\lambda^{2} /\left[\mu \mathrm{m}^{2}\right] \cdot I /\left(1.37 \cdot 10^{18}\left[\mathrm{~W} / \mathrm{cm}^{2}\right]\right)} \approx 2.2$. The laser focus was aligned to the entrance aperture of a $z_{C}=2.5 \mathrm{~mm}$ long gas cell (acceleration stage) as shown in Fig. 1. The gas was a 95\% $\mathrm{He}, 5 \% \mathrm{~N}_{2}$ mixture in order to make use of ionization injection [35-40]. The optimal electron density for acceleration within the cell was measured to be $n_{\mathrm{e}, \mathrm{LWFA}}=(1.0 \pm 0.25) \times 10^{19} \mathrm{~cm}^{-3}$ using interferometry. The density was cross checked by measuring the plasma wavelength by transverse optical probing [41]. Typical electron spectra for our laser and plasma conditions show an energy between 100 and $150 \mathrm{MeV}$ and an energy spread down to $<20 \mathrm{MeV}$ with a total charge of up to a few pC. Electrons were only observed when using gas mixtures and not observed when using pure helium, indicating that all electrons were injected by ionisation injection. The laser intensity was adjusted to operate close to the injection threshold: The electron signal vanishes

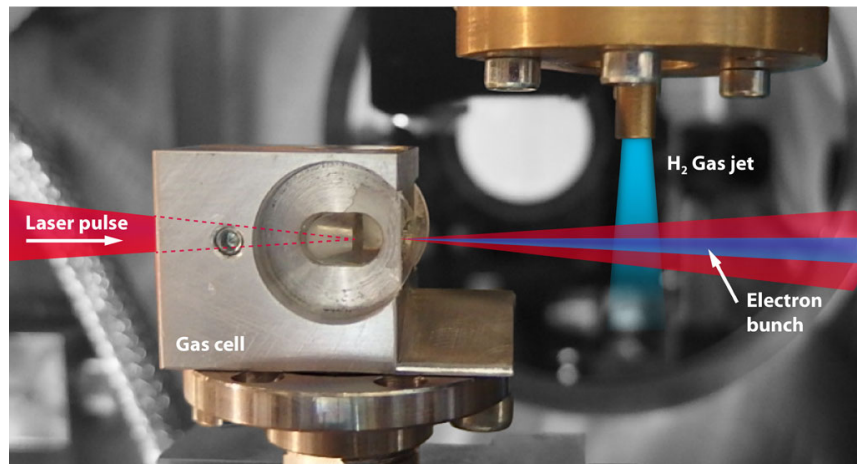

FIG. 1. Photograph of the experimental setup: the laser pulse, incident from the left, drives an LWFA stage in a mixed-species gas cell, and the generated electron beam expands in vacuum according to its divergence. If the second gas jet is turned on, the transmitted diverging laser pulse $\left(\theta_{\text {div }} \approx 80 \mathrm{mrad}\right)$ preionizes the $\mathrm{H}_{2}$ gas and the trailing electron beam $\left(\theta_{\text {div }} \approx 2.5 \mathrm{mrad}\right)$ experiences plasma lensing, which reduces the divergence substantially as observed on a scintillation screen (Figs. 2(a) and 2(b)). 
completely with a $10 \%$ reduction of laser energy making injection into any period other than the first wakefield period unlikely, thus constraining the temporal structure of the electron pulse to a single bunch. This is also supported by the elongation of the beam profile in polarization direction as it was shown by Mangles et al. [39]. After the acceleration stage, the laser propagated for a variable distance $L_{g}$ in vacuum before it traversed a supersonic $\mathrm{H}_{2}$ gas jet (lensing stage) of $2.5 \mathrm{~mm}$ length.

During the LWFA process, the laser's group velocity is reduced due to plasma dispersion and the electrons are being trapped in the plasma wake behind the laser pulse. The laser is still ahead of the electrons when it propagates through the second jet and ionizes the $\mathrm{H}_{2}$ gas. Hence, the electron bunch interacts with a preformed plasma in the lensing stage. For gap lengths $\left(L_{g}\right)$ between the LWFA stage and the lensing stage ranging from $8 \mathrm{~mm}$ to $24 \mathrm{~mm}$, ionization of $\mathrm{H} 2$ was clearly observed over the full length of the supersonic gas jet $(2.5 \mathrm{~mm})$ imaged using transverse optical probing. In contrast, ionization of pure He was not observed at any gap length, setting tight limits on the laser intensity in the gas jet.

We can therefore estimate an upper and lower bound for the laser intensity at the lensing stage. Using the Ammosov-Delone-Krainov (ADK) ionization rates [42] and assuming an ionization rate of $0.5 / \mathrm{fs}$, an upper bound of $I<1.75 \times 10^{15} \mathrm{~W} / \mathrm{cm}^{2}$ is found for $L_{g}=8.75 \mathrm{~mm}$ and a lower bound of $I>2.25 \times 10^{14} \mathrm{~W} / \mathrm{cm}^{2}$ is estimated at $L_{g}=24 \mathrm{~mm}$. This is in good agreement with the experimental conditions: Since the laser was guided with a matched spot size [24], we can assume that the divergence of the laser at the exit of the gas cell is similar to the focusing angle $(f / 12)$. Consequently the intensity will drop to $\approx 1 / 2000\left(L_{g}=8.75 \mathrm{~mm}\right)$ or $\approx 1 / 13000$ $\left(L_{g}=24 \mathrm{~mm}\right)$ compared to the intensity at the gas cell exit. Assuming a transmission of $\approx 30 \%$ the calculation matches the observed results. Note that this is a simplified approximation since the pulse becomes temporally compressed during its propagation in the gas cell [43]. It is likely, that the plasma gradient at the end of the gas cell reduces the laser's divergence, thus leaving a wider margin for the transmission.

The electron beam profile was measured both with and without the lensing stage using a scintillation screen at $z=57 \mathrm{~cm}$ after the position of the laser's vacuum focus. The multishot, averaged beam profiles recorded with this arrangement are shown in Figs. 2(a) and 2(b). The averages were obtained by aligning each image to the center of the electron beam for each laser shot before summing the images. The profile shown here is therefore only dependent on the beam divergence and is insensitive to shot-to-shot pointing fluctuations ( $4 \mathrm{mrad} \mathrm{rms}$ ). The solid angle covered by the electron beam was calculated by fitting a $2 \mathrm{D}$ Gaussian distribution to the beam profile and calculating the solid angle based on its standard deviation ellipse.

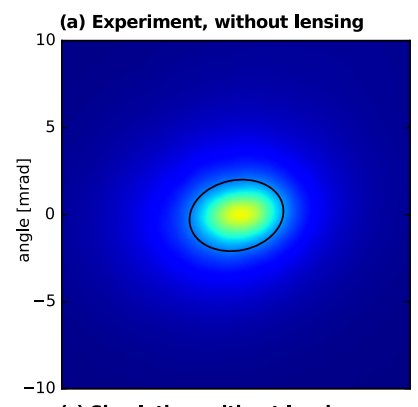

(b) Experiment, with lensing
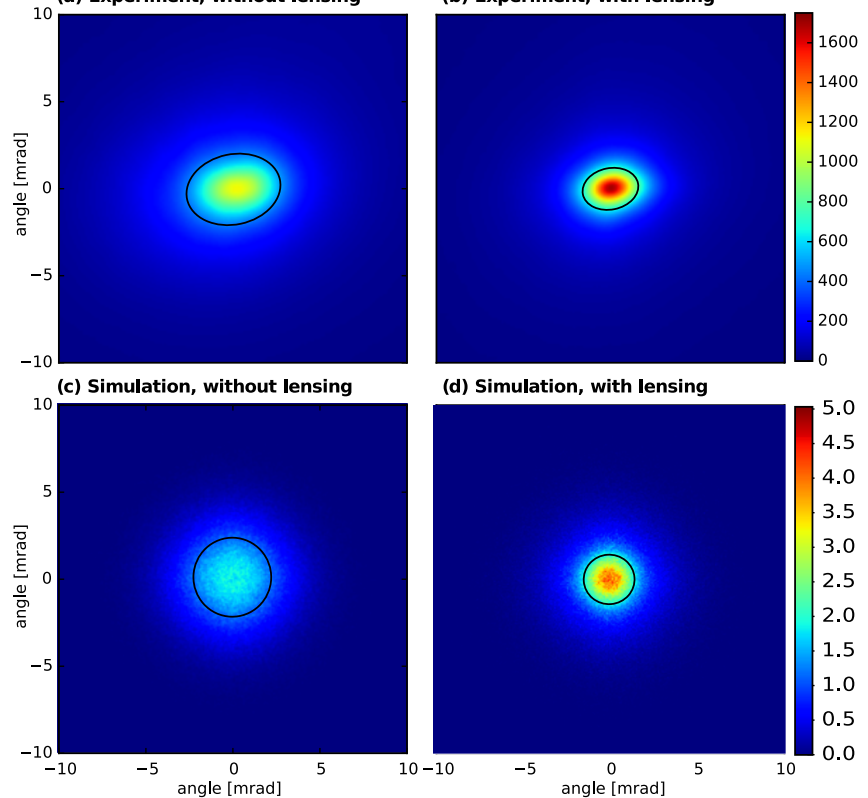

(d) Simulation, with lensing

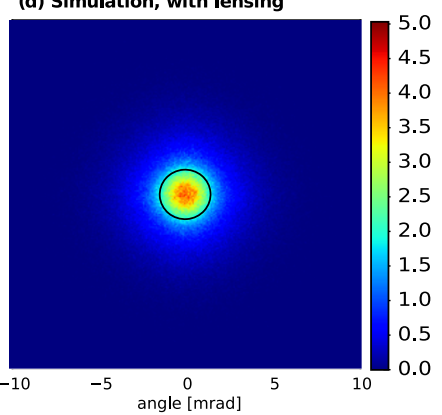

FIG. 2. Beam profiles as seen on the scintillation screen: Inset (a) shows the averaged and center aligned beam profile of 227 reference shots with the acceleration stage only. Inset (b) shows the averaged and center aligned beam profile of 175 shots with the focusing stage using an electron density of $1.6 \times 10^{19} \mathrm{~cm}^{-3}$ and a separation of $L_{g}=8.75 \mathrm{~mm}$. In both pictures the black ellipse encloses the standard deviation of a 2D gaussian fit function to the beam profile. The black ellipse reduces its area from $16 \mu \mathrm{sr}$ (left) to $6 \mu \mathrm{sr}$ (right) as the focusing stage is turned on. (c) and (d) show the corresponding data from the EPOCH3D Simulation: The bunch's initial divergence is set up to match the experimentally measured divergence of $16 \mu \mathrm{sr}$ (c). The encircled solid angle reduces to $7.3 \mu \mathrm{sr}$ after propagation through $2.5 \mathrm{~mm}$ plasma with a density of $n_{p}=1 \times 10^{19} / \mathrm{cm}^{3}$ with $0.5 \mathrm{~mm}$ linear ramps on both sides.

Figure 2 shows these ellipses drawn on the beam profiles without (a) and with (b) the lensing stage in operation. The decrease in solid angle and the increase of electron signal in the beam profile is clearly visible when the lensing stage was switched on. The average solid angle reduces from $16 \mu \mathrm{sr}$ to $6 \mu \mathrm{sr}$ for an electron density of $1.6 \times 10^{19} \mathrm{~cm}^{-3}$ in the lensing stage.

The dependence of the electron beam's solid angle on the plasma density in the lensing stage is shown in Fig. 3. It can be seen that increasing the plasma density in the lensing stage leads to a rapid reduction in solid angle of the accelerated electrons (blue). This is in agreement with a commensurate increase in the beam fluence recorded on the scintillation screen (red). For higher densities beyond $6 \times 10^{18} \mathrm{~cm}^{-3}$, the beam divergence continues to reduce more slowly, the beam intensity saturates, and the bunch charge in the central profile appears to reduce to $75 \%$ on average.

A single shot evaluation of the data set using the same methodology is shown in Fig. 4. Each dot represents a 


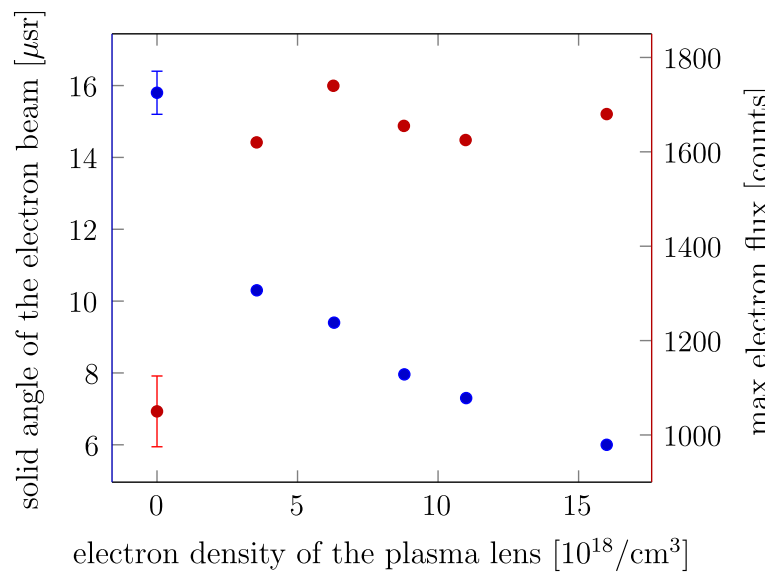

FIG. 3. Solid angle and maximum electron flux depending on the density of the focusing plasma: The solid angle (left axis, blue) of the electron beam decreases as the plasma density increases. The gap length was kept constant at $L_{g}=8.75 \mathrm{~mm}$ for all measurements. Each point is calculated from an averaged and center-aligned (see text) beam profile over 150 to 250 shots. The reference with the lensing stage turned off (density $=0$ ) has been measured at the beginning, middle and end of the scan. The error bars indicate the standard deviation $( \pm 1 \sigma)$ of these measurements. The error is similar for all measurements. The density measurements were taken using a wavefront sensor, resulting in an error of $\pm 0.5 \times 10^{19} / \mathrm{cm}^{3}$. The red curve (right axis) shows the peak electron flux on the scintillation screen.

single laser shot with (black) or without (green) the lensing stage using a plasma density of $1.6 \times 10^{19} / \mathrm{cm}^{3}$. The red arrow indicates the values obtained from the analysis of the center-aligned and averaged pictures [Figs. 2(a) and 2(b)] showing a consistent result with the moving point cloud. The solid angle decreases on average by a fixed factor due to the influence of the plasma lens, corresponding to a change in the gradient of the best fit. The slight reduction of the bunch charge (the charge within the standard deviation ellipse) to $75 \%$ on average is also visible as an increased number of shots at lower charge along the black line. Please note that this is not equivalent to a loss of total charge. In conclusion, the experimental results show that the electron bunch is focused in the second gas jet.

We want to stress that active plasma lensing can be excluded for our experimental conditions. This is due to the fact that the intensity of the laser in the second gas jet is too low to drive a wake field. The intensity can be estimated to be even lower than $I<1.7 \times 10^{15} \mathrm{~W} / \mathrm{cm}^{2}\left(a_{0}<0.029\right)$ as we were not able to ionize Helium in the second jet. The maximum plasma wake amplitude, which can be driven by such a transmitted and diverged laser pulse, is $\frac{\Delta n}{n}=a_{0}^{2}<10^{-3}$. This is much smaller than the amplitude of wake fields used for active focusing [34]. Furthermore, the laser has already significantly diverged when entering the second gas stage while the electron bunch remains much more collimated. Accordingly, the laser can be
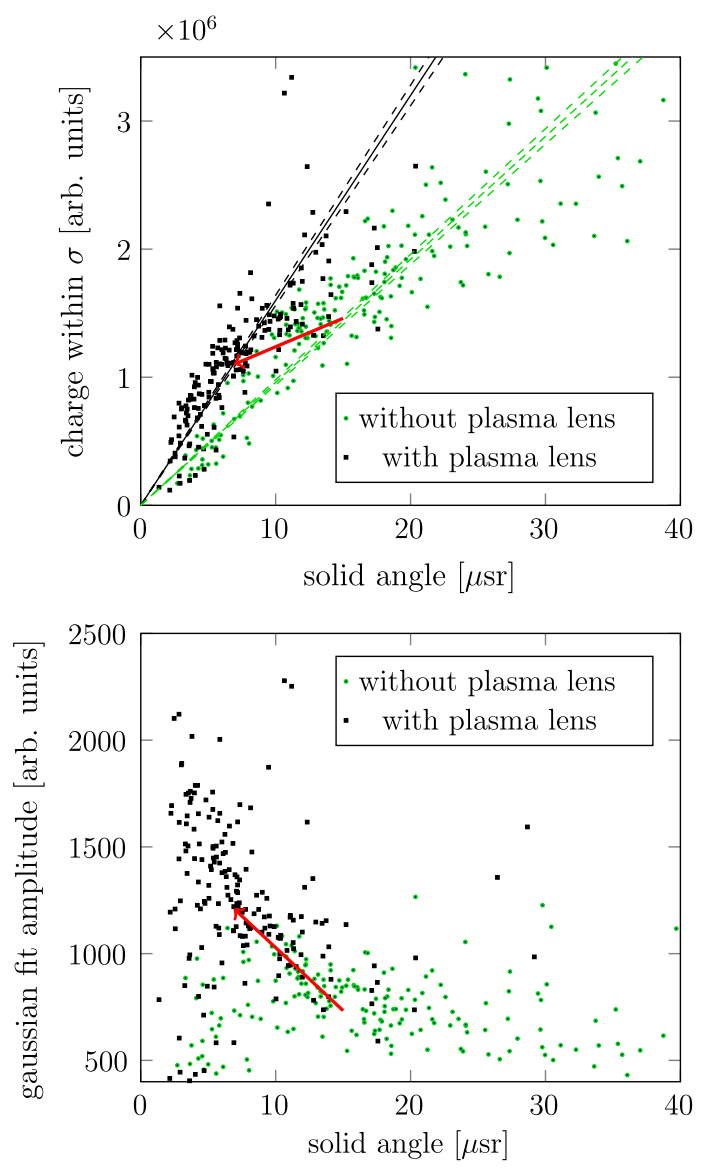

FIG. 4. Single shot data analysis of the plasma lensing effect: Each dot represents a single laser shot without the plasma lens (green) or with the plasma lens in operation (black). The shift of the point clouds is clearly visible. The same data sets are shown in Figs. 2(a) (green, no plasma lens) and 2(b) (black, plasma lens density $1.6 \times 10^{19} / \mathrm{cm}^{3}$ ). The red arrows therefore indicate the values obtained from the averaged analysis in Fig. 2. The gap length was constant at $L_{g}=8.75 \mathrm{~mm}$ Top: As the average solid angle reduces from $16 \mu \mathrm{sr}$ to $6.8 \mu \mathrm{sr}$, the charge in the beam's central region drops to $75 \%$. A linear fit reveals the increase of the beam's angular fluence from $(96 \pm 2) \times 10^{3}$ [a.u.] to $(160 \pm 4) \times 10^{3}$ [a.u.] with the lensing stage enabled. The dashed lines show the standard deviation of the fit. Bottom: Together with the reduction of the solid angle an increase in the amplitude of the 2D Gaussian fit by a factor of 1.65 is observed.

considered as a plane wave on the scale of the transverse electron bunch size. However, a plane wave can only induce longitudinal fields in the plasma due to symmetry. Since transverse fields are required for focusing, the effect of focusing is due to the reaction of the plasma to the charge of the electron bunch. To confirm this conclusion, we performed 3D particle-in-cell (PIC) simulations using the EPOCH3D [44] v4.3.7 code showing that the electron beam is self-focused while passing through a plasma without the aid of a laser driven plasma wake (passive lensing). The simulation parameters were adjusted to match the experimental conditions to our best knowledge. We set up a 
simulation box of $14 \times 200 \times 200 \mu \mathrm{m}^{3}$ with a resolution of $0.25 \mu \mathrm{m}$ in any spatial dimension. An electron bunch was initialized inside the box with $\gamma=\sqrt{1+\left(\frac{p}{m_{e} c}\right)^{2}}=160$ and a rms length of $\sigma_{x}=2 \mu \mathrm{m}$ and an rms width of $\sigma_{y, z}=5 \mu \mathrm{m}$ carrying a total charge of $Q=9 \mathrm{pC}$ sampled by $2 \times 10^{6}$ macro particles of variable weights. The transverse temperature of the bunch was adjusted such that its initial divergence matches the divergence measured in the experiment $(16 \mu \mathrm{sr})$. These bunch parameters lead to a normalized emittance $\epsilon_{N} \approx 1.9 \mathrm{~mm}$ mrad which is within the range of reported emittances, e.g., in Sears et al. [5] who reported a normalized emittance of $2.3 \pi \mathrm{mm} \mathrm{mrad}$ for LWFA electrons or Brunetti et al. [6] reporting $2.2 \pi \mathrm{mmmrad}$ on average, $1.1 \pi \mathrm{mmmrad}$ at best. Throughout the simulation, the bunch was kept centered within the box by moving the simulation box along with the bunch. The simulation results are shown in Figs. 2(c) and 2(d). We note that we used exactly the same fitting routine to deduce the electron beam divergence from the PIC simulation that we used to measure the divergence in the experimental data set. After a propagation distance of $x=2.5 \mathrm{~mm}$ through the plasma, the solid angle covered by the electrons decreased to $7.2 \mu \mathrm{sr}$ [Fig. 2(d)]. This value is comparable to the $6 \mu \mathrm{sr}$ measured in the experiment and thus the simulation reproduces the experimental result. However, the simulation strongly depends on the exact bunch parameters, particularly its longitudinal size, which are not directly accessible in the experiment.

While the simulations show that the lensing depends on the bunch parameters, the question arises whether the plasma lens amplifies the shot-to-shot variations significantly. It can be seen in Fig. 4 that the point clouds with and without the plasma lens occupy areas of similar size. Compared to the fluctuations of the laser-wakefield accelerated electron beam, the process of passive plasma lensing is robust with regards to fluctuations of the beam charge and initial divergence. The stability of our final electron beam is thus defined by the accelerator, not the passive plasma lens. While Fig. 3 suggests that further reductions of the beam divergence are anticipated at higher densities in the plasma lens stage, the shallower reduction of the divergence suggest that we might be close to a minimum.

Although the emittance of the beam was not measured in our experiment, the divergence reduction from $2.3 \mathrm{mrad}$ down to $1.4 \mathrm{mrad}$ seems not too far from the emittance limit, due to the short gap between the acceleration and the lensing stages: Assuming a normalized emittance of $\epsilon_{N}=2.0 \mathrm{~mm} \mathrm{mrad}$, the virtual source (VS) size of the electrons is $\sigma_{r}^{V S}=\frac{\epsilon_{N}}{\gamma \sigma_{\theta}}=5.4 \mu \mathrm{m}$ (Please note that the virtual source size does not correspond to the actual source size inside the plasma wake). Further assuming a vacuum drift of $L=7 \mathrm{~mm}$ due to the downramp profile of the gas flowing out of the gas cell, the bunch size at the lens is $\sigma_{r}^{\text {at lens }}=\sigma_{r}^{V S}+L \cdot \sigma_{\theta}=21.5 \mu \mathrm{m}$. Ideal refocusing of the beam would then lead to an emittance limited divergence of $\sigma_{\theta}^{\min }=\frac{\epsilon_{n}}{\gamma \sigma_{r}^{\text {al lens }}}=0.6 \mathrm{mrad}$. The emittance growth due the plasma lens in our experiment would therefore be less than factor 2.3. We note, that a lower initial emittance, such as that reported in one experiment [45], where the measurement took place inside the plasma, would imply a larger emittance growth. In contrast the estimation above assumes that the electron bunch is perfectly focused on the detector. A residual divergence would imply a smaller emittance growth.

In addition, the experimental value is altered by the fact that the spectrum of the electron beam is broad. From our experimental results, we cannot state at this point what part of the emittance growth is due to nonuniform fields in the lens and which part is caused by the chromatic dependence of the lens. Using these values the bunch density can be estimated $n_{b} \approx 2 \times 10^{15} / \mathrm{cm}^{3}$ assuming a charge of $5 \mathrm{pC}$. The measurements and simulations are therefore in the overdense regime $\left(n_{b} \ll n_{p}\right)$ and the electrons are expected to be focused by the magnetic part of the Lorentz force as described in the Introduction. In order to visualize the focusing effect of the passive plasma lens the force fields acting on the bunch are obtained from the 3D simulation. Figure 5 shows the forces acting on a central slice $(z=0)$ through the bunch. The bunch is moving into $+x$ direction and the color scale indicates the y-component of the Lorentz force $F_{L y}=q_{e}\left(E_{y}-c B_{z}\right)$. The focusing force in the simulation is indeed caused by the magnetic part of the Lorentz force as expected for focusing in the overdense regime. The strongest focusing fields are present in the tail of the electron bunch, however the center of the bunch still experiences a significant amount of focusing. A detailed analysis of the focusing forces in passive plasma lensing will be the subject of a separate publication.

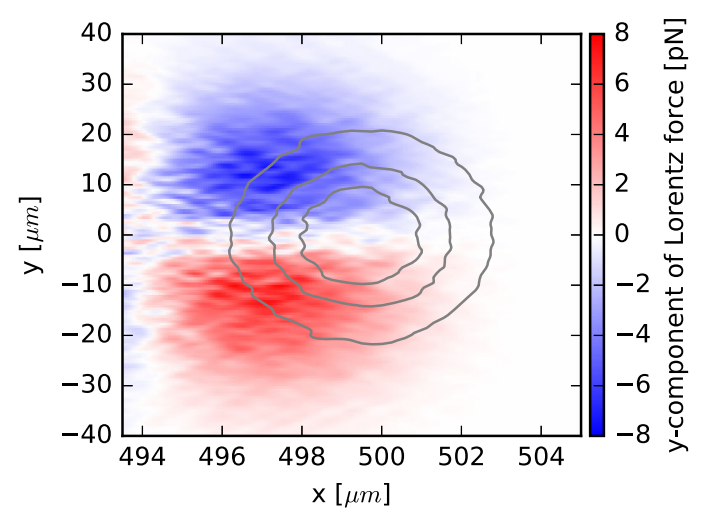

FIG. 5. The Lorentz force focusing the electron bunch: A central slice of the 3D simulation data is shown along $z=0$ after a propagation of $500 \mu \mathrm{m}$ through the plasma. The gray contour lines indicate $1 / \sqrt{2}, 1 / 2$ and $1 / 4$ of the maximum bunch density. The electron bunch is moving towards $+x$ direction and experiences a focusing Lorentz force (color scale). Although the strongest fields are present in the tail of the bunch, the first half of it still experiences a significant amount of focusing. 

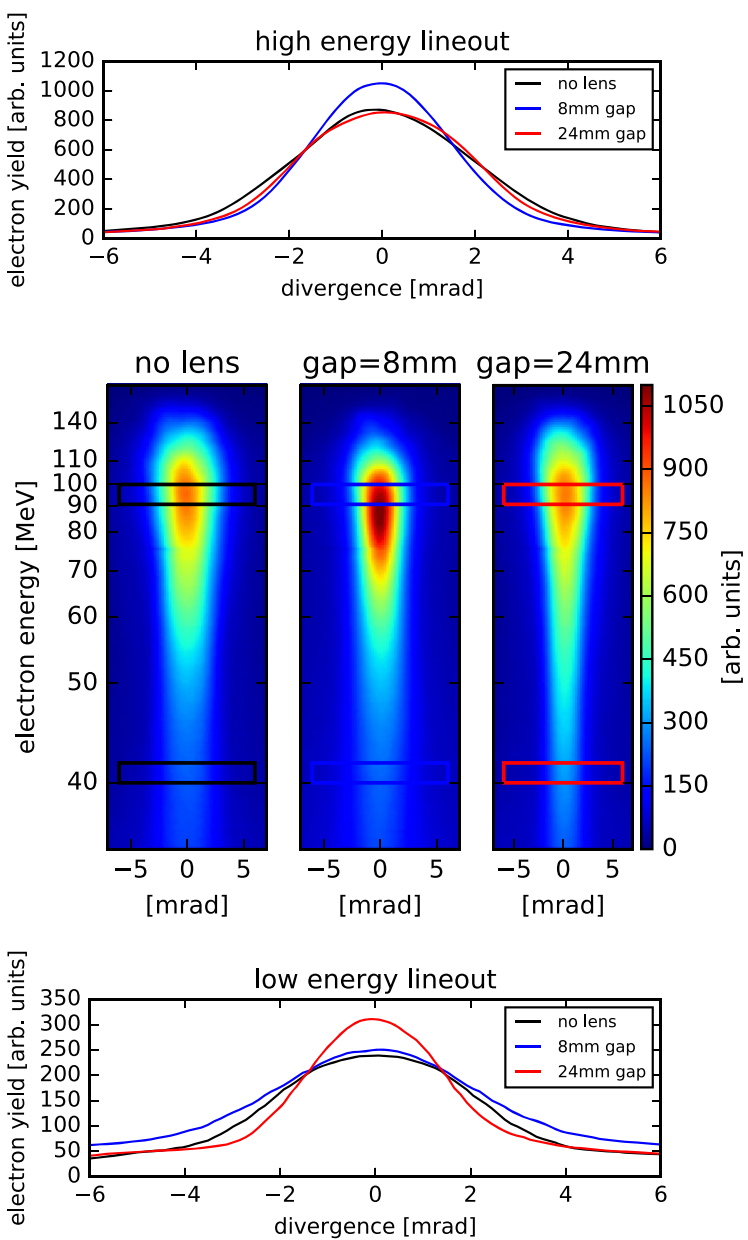

FIG. 6. Spectral dependence of the plasma lens: Each of the three spectra is aligned and averaged over 40-50 shots. The spectra show a comparison between the divergence of the electrons without (left) and with plasma lens (middle and right). The plasma lens uses an electron density of $(1.2 \pm 0.8) \times 10^{19} \mathrm{~cm}^{-3}$. Profiles of the high energy part (90-100 MeV) and the low energy part (40-42 MeV) are shown on top and bottom. It is shown that the high energy electrons are focused at $8 \mathrm{~mm}$ gap length (blue), whereas the low energetic electrons are focused at $24 \mathrm{~mm}$ gap length (red).

After obtaining the presented divergence data the setup was modified by inserting a magnet before the scintillation screen. This allowed us to resolve the final electron spectrum, while still maintaining divergence information in the other (nondispersive) direction. In the analysis procedure, every single shot data was aligned in the divergence direction before averaging to ensure that the averaged picture is dominated by the divergence of the beam and not its pointing stability. For each position, we were averaging between 40 and 50 shots. The averaged and aligned scintillation screen pictures are shown in Fig. 6 for two different gap lengths with lensing and a reference case without lensing for comparison. The focusing effect is clearly visible in two features: First the reduction in
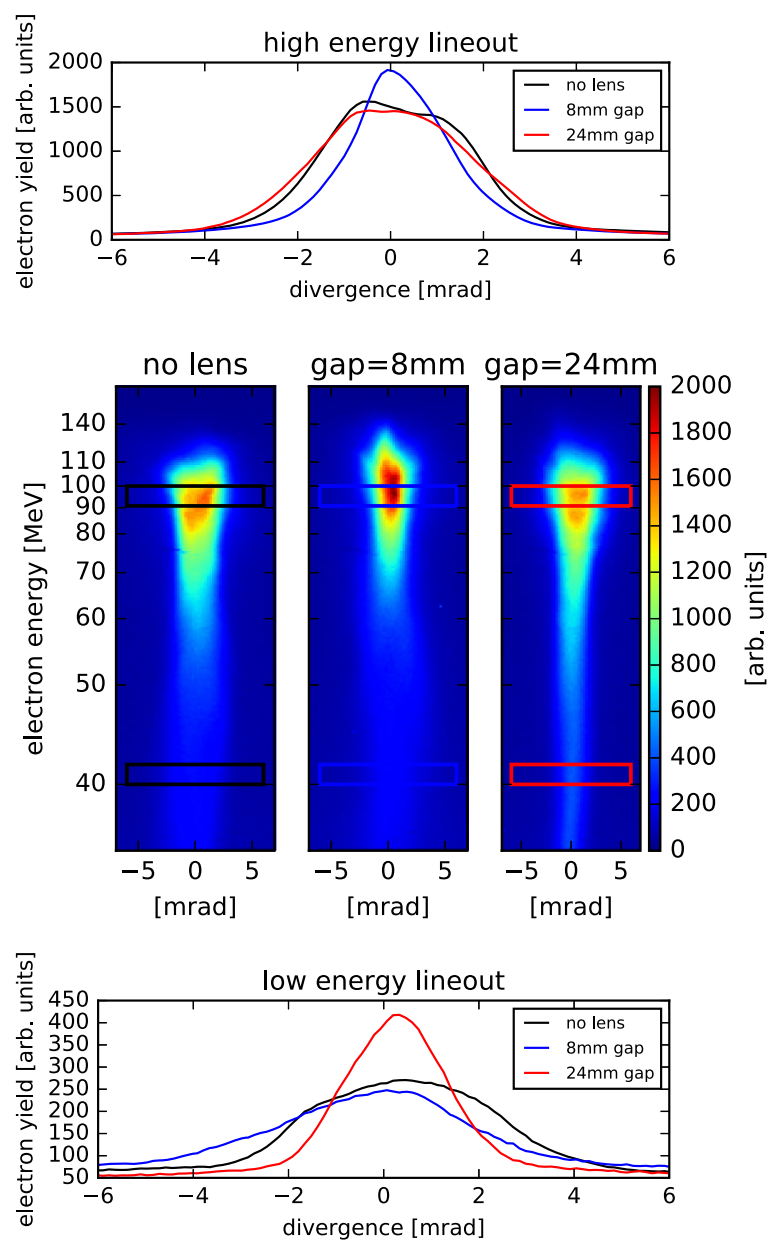

FIG. 7. Spectral dependence of the lens for three single shots: The plots are equal to Fig. 6, but with only three single shots presented. The same behavior as in Fig. 6 can be observed: The high energy electrons are focused at $8 \mathrm{~mm}$ gap length (blue) whereas the low energetic electrons are focused at $24 \mathrm{~mm}$ gap length (red). This shows that the effect of plasma lensing can be observed in the single-shot data and the averaged data.

divergence and second the increase in charge density on the scintillation screen. At $8 \mathrm{~mm}$ gap length, the electrons around $95 \mathrm{MeV}$ show a reduced divergence compared to the reference shots as well as increased signal intensity on the beam axis (profile at high energies in Fig. 6). At $24 \mathrm{~mm}$ gap length, the same holds true for the electrons around $40 \mathrm{MeV}$ (profile at low energies in Fig. 6). We note, that the spectral data (Figs. 6, 7 and 8) and the profile data (Figs. 2, 3 and 4) have been recorded during different parts of the experimental campaign. This might be the reason for a slightly different reduction in divergence, that is shown in the profile data.

In order to investigate the spectral dependence of the passive plasma lensing effect further, the ratios between lens and reference shots for both, divergence reduction and relative intensity are visualized in Fig. 8 for 5 different gap lengths. It is very important to cross check that the plasma 
Divergence reduction

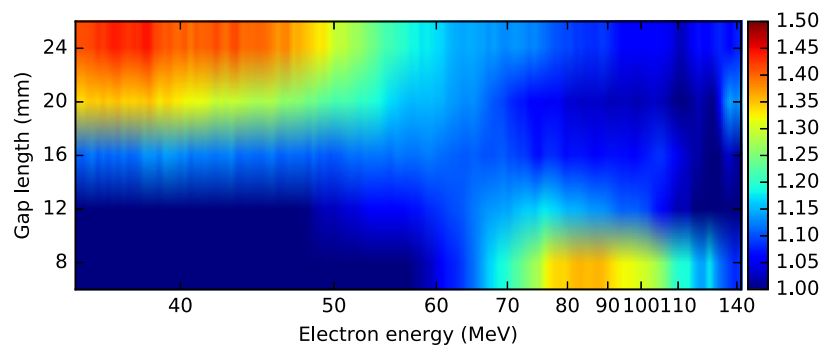

Intensity change

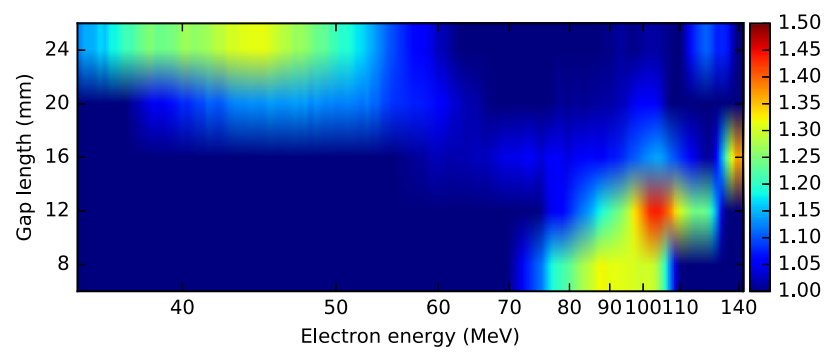

FIG. 8. The spectral dependence of the plasma lens measured with five different gap lengths: The color maps show the divergence reduction $\left(=\theta_{\mathrm{div}}^{\text {without lens }} / \theta_{\mathrm{div}}^{\text {with lens }}\right.$, upper panel) and the relative intensity change on the lanex screen (lower panel). Both measurements are in good agreement, since a divergence reduction is followed by an increase in spectral intensity. The data is obtained by an average over 40-50 shots for each gap length using the same data set as in Fig. 6.

lens is visible in the reduction of the electron divergence as well as the increase of electron density on the scintillation screen, otherwise a reduced angular acceptance could cause an apparent lensing effect. As seen in Fig. 8 both measurements are in good agreement: For small gap length $(<12 \mathrm{~mm})$ the plasma lens effect is dominant in the high energy part of the spectrum between 80 and $130 \mathrm{MeV}$. With increasing distance between the accelerator and the lensing stage the focusing effect acts more strongly on the low energy electrons around $70 \mathrm{MeV}$ and below. However, the plasma lens effect is a complicated interaction depending on many parameters such as bunch charge, density, gap length and temporal structure of the bunch.

\section{CONCLUSION}

In conclusion, we have demonstrated a compact, passive plasma lens in a simple setup using LWFA electrons. The solid angle of the accelerated electron bunch decreases due to the lensing by more than a factor of 2 . This is in line with an increase of the fluence of the electron beam, which is often a crucial parameter for experiments and applications. Our experimental data show that the angular reduction due the plasma lens is stable with regards to bunch charge fluctuations from the LWFA accelerator and tunable with respect to electron energy making the passive plasma lens a highly useful and robust device for controlling the divergence of LWFA beams. The measured decrease in divergence is in good agreement with 3D PIC simulations using the experimental parameters. In addition, the repetition rate of the passive plasma lens is not limited to the repetition rate of any external driver due to its passive character. Therefore it is generally suited to focus electron bunches at high repetition rates that will be available soon with the LCLS-II / FACET-II and the European XFEL.

\section{ACKNOWLEDGMENTS}

B. Beleites and F. Ronneberger have contributed to this work by operating the JETI laser facility. W. Ziegler made significant contributions to the gas cell design. The authors gratefully acknowledge the following open-sourceprojects: Numpy [46], Matplotlib [47], IPython [48], postpic [49]. The authors gratefully acknowledge the computing time granted by the John von Neumann Institute for Computing (NIC) and provided on the supercomputer JUROPA at Jülich Supercomputing Centre (JSC). This study was supported by DFG (Grants No. TR18-B9, and No. KA 2869/2-1), BMBF (Contracts No. 05K10SJ2, No. 03ZIK052, and No. 05K16SJC), and the European Regional Development Fund (EFRE).

[1] E. Esarey, C. B. Schroeder, and W. P. Leemans, Physics of laser-driven plasma-based electron accelerators, Rev. Mod. Phys. 81, 1229 (2009).

[2] X. Wang, R. Zgadzaj, N. Fazel, Z. Li, S. A. Yi, X. Zhang, W. Henderson, Y-Y. Chang, R. Korzekwa, H.-E. Tsai et al., Quasi-monoenergetic laser-plasma acceleration of electrons to $2 \mathrm{GeV}$, Nat. Commun. 4, 1988 (2013).

[3] H. T. Kim, K. H. Pae, H. J. Cha, I. J. Kim, T. J. Yu, J. H. Sung, S. K. Lee, T. M. Jeong, and J. Lee, Enhancement of Electron Energy to the Multi-GeV Regime by a Dual-Stage Laser-Wakefield Accelerator Pumped by Petawatt Laser Pulses, Phys. Rev. Lett. 111, 165002 (2013).

[4] W. P. Leemans, A. J. Gonsalves, H-S. Mao, K. Nakamura, C. Benedetti, C. B. Schroeder, C. S. Tóth, J. Daniels, D. E. Mittelberger, S.S. Bulanov et al., Multi-GeV Electron Beams from Capillary-Discharge-Guided Subpetawatt Laser Pulses in the Self-Trapping Regime, Phys. Rev. Lett. 113, 245002 (2014).

[5] C. M. S. Sears, A. Buck, K. Schmid, J. Mikhailova, F. Krausz, and L. Veisz, Emittance and divergence of laser wakefield accelerated electrons, Phys. Rev. ST Accel. Beams 13, 092803 (2010).

[6] E. Brunetti, R. P. Shanks, G. G. Manahan, M. R. Islam, B. Ersfeld, M. P. Anania, S. Cipiccia, R. C. Issac, G. Raj, G. Vieux, G. H. Welsh, S. M. Wiggins, and D. A. Jaroszynski, Low Emittance, High Brilliance Relativistic Electron Beams from a Laser-Plasma Accelerator, Phys. Rev. Lett. 105, 215007 (2010).

[7] G. Hairapetian, P. Davis, C. E. Clayton, C. Joshi, S. C. Hartman, C. Pellegrini, and T. Katsouleas, Experimental Demonstration of Dynamic Focusing of a Relativistic 
Electron Bunch by an Overdense Plasma Lens, Phys. Rev. Lett. 72, 2403 (1994).

[8] R. Govil, W. P. Leemans, E. Y. Backhaus, and J. S. Wurtele, Observation of Return Current Effects in a Passive Plasma Lens, Phys. Rev. Lett. 83, 3202 (1999).

[9] M. C. Thompson, H. Badakov, J. B. Rosenzweig, G. Travish, R. Fliller, G. M. Kazakevich, P. Piot, J. Santucci, $\mathrm{J}$. Li, and R. Tikhoplav, in Proceedings of the 22nd Particle Accelerator Conference, PAC-2007, Albuquerque, NM (IEEE, New York, 2007), p. 1907.

[10] H.-P. Schlenvoigt, K. Haupt, A. Debus, F. Budde, O. Jäckel, S. Pfotenhauer, H. Schwoerer, E. Rohwer, J. G. Gallacher, E. Brunetti et al., A compact synchrotron radiation source driven by a laser-plasma wakefield accelerator, Nat. Phys. 4, 130 (2008).

[11] M. Fuchs, R. Weingartner, A. Popp, Z. Major, S. Becker, J. Osterhoff, I. Cortrie, B. Zeitler, R. Hörlein, G. D. Tsakiris et al., Laser-driven soft-x-ray undulator source, Nat. Phys. 5, 826 (2009).

[12] C. Widmann, V. Afonso Rodriguez, S. Kuschel, M. Nicolai, R. Rossmanith, A. Sävert, M. B. Schwab, W. Werner, M. C. Kaluza, A. Bernhard et al., First tests of a beam transport system from laser wakefield accelerator to a transverse gradient undulator, in Proceedings of IPAC2015, Richmond, VA (2015).

[13] G. Sarri, W. Schumaker, A. Di Piazza, M. Vargas, B. Dromey, M. E. Dieckmann, V. Chvykov, A. Maksimchuk, V. Yanovsky, Z. H. He, B. X. Hou, J. A. Nees, A. G. R. Thomas, C. H. Keitel, M. Zepf, and K. Krushelnick, TableTop Laser-Based Source of Femtosecond, Collimated, Ultrarelativistic Positron Beams, Phys. Rev. Lett. 110, 255002 (2013).

[14] Y. Glinec, J. Faure, L. Le Dain, S. Darbon, T. Hosokai, J. J. Santos, E. Lefebvre, J.-P. Rousseau, F. Burgy, B. Mercier et al., High-Resolution $\gamma$-Ray Radiography Produced by a Laser-Plasma Driven Electron Source, Phys. Rev. Lett. 94, 025003 (2005).

[15] S. Corde, C. Thaury, K. Ta Phuoc, A. Lifschitz, G. Lambert, J. Faure, O. Lundh, E. Benveniste, A. Ben-Ismail, L. Arantchuk, A. Marciniak, A. Stordeur, P. Brijesh, A. Rousse, A. Specka, and V. Malka, Mapping the X-ray Emission Region in a Laser-Plasma Accelerator, Phys. Rev. Lett. 107, 215004 (2011).

[16] N. D. Powers, I. Ghebregziabher, G. Golovin, C. Liu, S. Chen, S. Banerjee, J. Zhang, and D. P. Umstadter, Quasimonoenergetic and tunable $\mathrm{X}$-rays from a laser-driven compton light source, Nat. Photonics 8, 28 (2014).

[17] G. Sarri, D. J. Corvan, W. Schumaker, J. M. Cole, A. Di Piazza, H. Ahmed, C. Harvey, C. H. Keitel, K. Krushelnick, S. P. D. Mangles, Z. Najmudin, D. Symes, A. G. R. Thomas, M. Yeung, Z. Zhao, and M. Zepf, Ultrahigh Brilliance Multi-MeV $\gamma$-Ray Beams from Nonlinear Relativistic Thomson Scattering, Phys. Rev. Lett. 113, 224801 (2014).

[18] K Khrennikov, J. Wenz, A. Buck, J. Xu, M. Heigoldt, L. Veisz, and S. Karsch, Tunable All-Optical Quasimonochromatic Thomson X-ray Source in the Nonlinear Regime, Phys. Rev. Lett. 114, 195003 (2015).

[19] J. Krall and G. Joyce, Transverse equilibrium and stability of the primary beam in the plasma wakefield accelerator, Phys. Plasmas 2, 1326 (1995).
[20] B. Hidding, T. Königstein, J. Osterholz, S. Karsch, O. Willi, and G. Pretzler, Monoenergetic Energy Doubling in a Hybrid Laser-Plasma Wakefield Accelerator, Phys. Rev. Lett. 104, 195002 (2010).

[21] M. Litos, E. Adli, W. An, C. I. Clarke, C. E. Clayton, S. Corde, J. P. Delahaye, R. J. England, A. S. Fisher, J. Frederico et al., High-efficiency acceleration of an electron beam in a plasma wakefield accelerator, Nature (London) 515, 92 (2014).

[22] P. E. Masson-Laborde, M. Z. Mo, A. Ali, S. Fourmaux, P. Lassonde, J. C. Kieffer, W. Rozmus, D. Teychenné, and R. Fedosejevs, Giga-electronvolt electrons due to a transition from laser wakefield acceleration to plasma wakefield acceleration, Phys. Plasmas 21, 123113 (2014).

[23] B. Hidding, G. Pretzler, J. B. Rosenzweig, T. Königstein, D. Schiller, and D. L. Bruhwiler, Ultracold Electron Bunch Generation via Plasma Photocathode Emission and Acceleration in a Beam-Driven Plasma Blowout, Phys. Rev. Lett. 108, 035001 (2012).

[24] Wei Lu, M. Tzoufras, C. Joshi, F. S. Tsung, W. B. Mori, J. Vieira, R. A. Fonseca, and L. O. Silva, Generating multi-GeV electron bunches using single stage laser wakefield acceleration in a 3D nonlinear regime, Phys. Rev. ST Accel. Beams 10, 061301 (2007).

[25] J. J. Su, T. Katsouleas, J. M. Dawson, and R. Fedele, Plasma lenses for focusing particle beams, Phys. Rev. A 41, 3321 (1990).

[26] P. Chen, A possible final focusing mechanism for linear colliders, Part. Accel. 20, 171 (1987).

[27] J. B. Rosenzweig, P. Schoessow, B. Cole, C. Ho, W. Gai, R. Konecny, S. Mtingwa, J. Norem, M. Rosing, and J. Simpson, Demonstration of electron beam self-focusing in plasma wake fields, Phys. Fluids B 2, 1376 (1990).

[28] H. Nakanishi, Y. Yoshida, T. Ueda, T. Kozawa, H. Shibata, K. Nakajima, T. Kurihara, N. Yugami, Y. Nishida, T. Kobayashi, A. Enomoto, T. Oogoe, H. Kobayashi, B. S. Newberger, S. Tagawa, K. Miya, and A. Ogata, Direct Observation of Plasma-Lens Effect, Phys. Rev. Lett. 66, 1870 (1991).

[29] G. Hairapetian, P. Davis, C. E. Clayton, C. Joshi, C. Pellegrini, and T. Katsouleas, Transverse dynamics of a short, relativistic electron bunch in a plasma lens, Phys. Plasmas 2, 2555 (1995).

[30] R. Govil, S. J. Wheeler, and W. P. Leemans, in Proceedings of the Particle Accelerator Conference, Vancouver, BC, 1997 (IEEE, New York, 1997), Vol. 1, p. 654.

[31] A. Buck, M. Nicolai, K. Schmid, C. M. S. Sears, A. Sävert, J. M. Mikhailova, F. Krausz, M. C. Kaluza, and L. Veisz, Real-time observation of laser-driven electron acceleration, Nat. Phys. 7, 543 (2011).

[32] J. van Tilborg, S. Steinke, C. G. R. Geddes, N. H. Matlis, B. H. Shaw, A. J. Gonsalves, J. V. Huijts, K. Nakamura, J. Daniels, C. B. Schroeder, C. Benedetti, E. Esarey, S. S. Bulanov, N. A. Bobrova, P. V. Sasorov, and W. P. Leemans, Active Plasma Lensing for Relativistic Laser-PlasmaAccelerated Electron Beams, Phys. Rev. Lett. 115, 184802 (2015).

[33] R. Lehe, C. Thaury, E. Guillaume, A. Lifschitz, and V. Malka, Laser-plasma lens for laser-wakefield accelerators, Phys. Rev. ST Accel. Beams 17, 121301 (2014). 
[34] C. Thaury, E. Guillaume, A. Döpp, R. Lehe, A. Lifschitz, K. Ta Phuoc, J. Gautier, J.-P. Goddet, A. Tafzi, A. Flacco et al., Demonstration of relativistic electron beam focusing by a laser-plasma lens, Nat. Commun. 6, 6860 (2015).

[35] M. Chen, Z.-M. Sheng, Y.-Y. Ma, and J. Zhang, Electron injection and trapping in a laser wakefield by field ionization to high-charge states of gases, J. Appl. Phys. 99, 056109 (2006).

[36] A. Pak, K. A. Marsh, S. F. Martins, W. Lu, W. B. Mori, and C. Joshi, Injection and Trapping of Tunnel-Ionized Electrons into Laser-Produced Wakes, Phys. Rev. Lett. 104, 025003 (2010).

[37] C. McGuffey, A. G. R. Thomas, W. Schumaker, T. Matsuoka, V. Chvykov, F. J. Dollar, G. Kalintchenko, V. Yanovsky, A. Maksimchuk, K. Krushelnick, V. Y. Bychenkov, I. V. Glazyrin, and A. V. Karpeev, Ionization Induced Trapping in a Laser Wakefield Accelerator, Phys. Rev. Lett. 104, 025004 (2010).

[38] C. E. Clayton, J. E. Ralph, F. Albert, R. A. Fonseca, S. H. Glenzer, C. Joshi, W. Lu, K. A. Marsh, S. F. Martins, W. B. Mori, A. Pak, F. S. Tsung, B. B. Pollock, J. S. Ross, L. O. Silva, and D. H. Froula, Self-Guided Laser Wakefield Acceleration beyond $1 \mathrm{GeV}$ Using Ionization-Induced Injection, Phys. Rev. Lett. 105, 105003 (2010).

[39] S. P. D. Mangles, A. G. R. Thomas, M. C. Kaluza, O. Lundh, F. Lindau, A. Persson, F. S. Tsung, Z. Najmudin, W. B. Mori, C.-G. Wahlström, and K. Krushelnick, LaserWakefield Acceleration of Monoenergetic Electron Beams in the First Plasma-Wave Period, Phys. Rev. Lett. 96, 215001 (2006).

[40] M. Chen, E. Esarey, C. B. Schroeder, C. G. R. Geddes, and W. P. Leemans, Theory of ionization-induced trapping in laser-plasma accelerators, Phys. Plasmas (1994-present) 19, 033101 (2012).
[41] M. B. Schwab, A. Sävert, O. Jäckel, J. Polz, M. Schnell, T. Rinck, L. Veisz, M. Möller, P. Hansinger, G. G. Paulus et al., Few-cycle optical probe-pulse for investigation of relativistic laser-plasma interactions, Appl. Phys. Lett. 103, 191118 (2013).

[42] M. V. Ammosov, N. B. Delone, and V. P. Krainov, Tunnel ionization of complex atoms and atomic ions in electromagnetic field, JETP 64, 1191 (1986).

[43] J. Faure, Y. Glinec, J. J. Santos, F. Ewald, J.-P. Rousseau, S. Kiselev, A. Pukhov, T. Hosokai, and V. Malka, Observation of Laser-Pulse Shortening in Nonlinear Plasma Waves, Phys. Rev. Lett. 95, 205003 (2005).

[44] T. D. Arber, K. Bennett, C.S. Brady, A. LawrenceDouglas, M. G. Ramsay, N. J. Sircombe, P. Gillies, R. G. Evans, H. Schmitz, A. R. Bell, and C. P. Ridgers, Contemporary particle-in-cell approach to laser-plasma modelling, Plasma Phys. Controlled Fusion 57, 113001 (2015).

[45] G. R. Plateau, C. G. R. Geddes, D. B. Thorn, M. Chen, C. Benedetti, E. Esarey, A. J. Gonsalves, N. H. Matlis, K. Nakamura, C. B. Schroeder, S. Shiraishi, T. Sokollik, J. van Tilborg, Cs. Toth, S. Trotsenko, T. S. Kim, M. Battaglia, Th. Stöhlker, and W. P. Leemans, Low-Emittance Electron Bunches from a Laser-Plasma Accelerator Measured Using Single-Shot X-ray Spectroscopy, Phys. Rev. Lett. 109, 064802 (2012).

[46] S. van der Walt, S. Chris Colbert, and G. Varoquaux, The numpy array: A structure for efficient numerical computation, Comput. Sci. Eng. 13, 22 (2011).

[47] John D. Hunter, Matplotlib: A 2D graphics environment, Comput. Sci. Eng. 9, 90 (2007).

[48] F. Pérez and B. E. Granger, Ipython: A system for interactive scientific computing, Comput. Sci. Eng. 9, 21 (2007).

[49] S. Kuschel, Postpic: The open-source particle in cell postprocessor (2015), http://github.com/skuschel/postpic. 